\title{
Synchronization Control for Nonlinear Stochastic Dynamical Networks: Pinning Impulsive Strategy
}

\author{
Jianquan Lu, Jürgen Kurths, Jinde Cao, Senior Member, IEEE, Nariman Mahdavi, and Chi Huang
}

\begin{abstract}
In this paper, a new control strategy is proposed for the synchronization of stochastic dynamical networks with nonlinear coupling. Pinning state feedback controllers have been proved to be effective for synchronization control of statecoupled dynamical networks. We will show that pinning impulsive controllers are also effective for synchronization control of the above mentioned dynamical networks. Some generic mean square stability criteria are derived in terms of algebraic conditions, which guarantee that the whole state-coupled dynamical network can be forced to some desired trajectory by placing impulsive controllers on a small fraction of nodes. An effective method is given to select the nodes which should be controlled at each impulsive constants. The proportion of the controlled nodes guaranteeing the stability is explicitly obtained, and the synchronization region is also derived and clearly plotted. Numerical simulations are exploited to demonstrate the effectiveness of the pinning impulsive strategy proposed in this paper.
\end{abstract}

Index Terms-Nonlinear coupling, pinning impulsive control, state-coupled dynamical network, synchronization.

\section{INTRODUCTION}

$\mathbf{C}$ OMPLEX dynamical networks are composed of a large number of interconnected dynamical nodes, in which each node is a unit with specific contents [1]-[3]. Typical

Manuscript received August 8, 2011; revised October 26, 2011; accepted December 3, 2011. Date of publication January 6, 2012; date of current version February 8, 2012. The work of J. Q. Lu was supported by the National Natural Science Foundation of China under Grant 61175119, the Natural Science Foundation of the Jiangsu Province of China under Grant BK2010408, the Program for New Century Excellent Talents in University, under Project NCET-10-0329, and the Alexander von Humboldt Foundation of Germany. The work of J. Kurths was supported by SUMO (EU), GSDP (EU), and ECONS (WGL). The work of J. D. Cao was supported by the National Natural Science Foundation of China under Grant 11072059, the Specialized Research Fund for the Doctoral Program of Higher Education under Grant 20070286003, and the Natural Science Foundation of the Jiangsu Province of China under Grant BK2009271.

J. Lu is with the Department of Mathematics, Southeast University, Nanjing 210096, China. He is also with the Potsdam Institute for Climate Impact Research, Potsdam D-14415, Germany (e-mail: jqluma@ seu.edu.cn).

J. Kurths is with the Potsdam Institute for Climate Impact Research, Potsdam D-14415, Germany. He is also with the Department of Physics, Humboldt University Berlin, Berlin 10117, Germany, and with the Institute for Complex Systems and Mathematical Biology, University of Aberdeen, Aberdeen AB24 3UE, U.K. (e-mail: Juergen.Kurths@pik-potsdam.de).

J. Cao is with the Department of Mathematics, Southeast University, Nanjing 210096, China (e-mail: jdcao@seu.edu.cn).

N. Mahdavi is with the Department of Electrical Engineering, Amirkabir University of Technology, Tehran 16846-13114, Iran. He is also with the Potsdam Institute for Climate Impact Research, Potsdam 14412, Germany (e-mail: nariman.mahdavi@gmail.com).

C. Huang is with the Department of Mathematics, City University of Hong Kong, Kowloon 2788, Hong Kong (e-mail: huangchima@gmail.com).

Color versions of one or more of the figures in this paper are available online at http://ieeexplore.ieee.org.

Digital Object Identifier 10.1109/TNNLS.2011.2179312 examples of complex networks include the Internet, the World Wide Web, neural networks, food webs, cellular and metabolic networks, etc., [4], [5]. Since the seminal papers on "smallworld" and "scale-free" properties [4], [6], complex networks have become a focus of research and have received increasing attention from various fields of science and engineering. Complex networks often exhibit complex and interesting dynamical behavior including synchronization [3], [7], consensus [8], flocking etc. As one of the most interesting and important collective behavior in dynamical networks, synchronization has attracted special attention of researchers in different fields [9]-[13].

Synchronization in dynamical networks is realized via a sufficient information exchange among the nodes' interconnections [8], [14], [15], which makes the final synchronous state difficult to predict. However, for many biological, physical and social dynamical networks, there exists a common requirement to regulate the behavior of large ensembles of interacting units. Some regulatory mechanisms have been uncovered in the context of biological, physiological, and cellular processes [16], which are fundamental to guarantee the correct functioning of the whole network. Examples include the control of the respiratory rhythm played by synaptically coupled pacemaker neurons in the medulla in physiology [17], and opinion leader in social networks. Hence, in many cases, controllers are necessary to be designed to force the unpredicted final synchronous state into a certain required objective trajectory [18]-[22].

It has been revealed that, in the process of controlling various networks, feedback control serves as a simple and effective strategy for stabilization and synchronization. Different kinds of effective methods, including adaptive controllers [23], [24], impulsive controllers [25]-[27] and pinning state feedback controllers [19], [20], have been designed for the stabilization and synchronization of complex dynamical networks. In [23], [24], the feedback strength is asymptotically enhanced according to a certain update law for the stabilization and synchronization of dynamical networks. In [26], distributed impulsive controllers are properly designed for the synchronization control of dynamical networks. Pinning state feedback controllers were first proposed to control multi-mode laser systems in [28], and have recently been used for the synchronization of complex networks by controlling a small fraction of nodes [19], [20], [22]. These methods have been shown to be effective for the synchronization control of networks.

For many realistic networks, the state of nodes is often subject to instantaneous perturbations and experience abrupt change at certain instants which may be caused by switching 
phenomena, frequency change or sudden noise, i.e., it exhibits impulsive effects. On the other hand, each individual node in dynamical networks is often subject to various types of noise and uncertainty, which can have a great influence on the behavior of dynamical networks. Impulsive control strategy has also been shown to be an effective control strategy in many fields due to its potential advantages over general continuous control schemes [26], [29]. However, in previous studies, when impulsive controllers are designed for the synchronization control of dynamical networks with state-coupling, all of the nodes should be controlled, which means that the controlling cost is very high. Pinning state feedback control, which means that only a small fraction of nodes is directly controlled, has been proved to be effective for the synchronization of dynamical networks with state-coupling. Then one may ask: 1) can the stochastic dynamical network be synchronized by impulsively controlling a small fraction of nodes; 2) that is, can we also design a certain pinning impulsive control strategy for the synchronization of stochastic dynamical networks; and 3 ) this paper is devoted to solving this problem. Some genetic criteria are given to judge whether dynamical networks can be globally exponentially forced to a desired equilibrium by impulsively controlling a small fraction of nodes. Numerical examples are finally given to demonstrate the effectiveness of the proposed impulsive strategy.

Notations: The standard notations will be used in this paper. $I_{n}$ is the identity matrix of order $n . \lambda_{\max }(\cdot)$ is used to denote the maximum eigenvalue of a real symmetric matrix. $\mathbb{R}^{n}$ denotes the $n$-dimensional Euclidean space. $\mathbb{R}^{n \times n}$ are $n \times n$ real matrices. $\|x\|$ denotes the Euclidean norm of vector $x \in \mathbb{R}^{n}$. Let $\mathbb{R}^{+}=[0,+\infty), \mathbb{N}=\{1,2,3, \ldots\}$. The superscript " $T$ " represents the transpose. For any random variable $\zeta$, let $E(\zeta)$ be the expectation value of $\zeta$. \#G denotes the number of elements of a finite set $G$.

\section{PRELIMINARIES}

In this paper, we consider the following stochastic dynamical network with nonlinear coupling:

$$
\begin{aligned}
d x_{i}(t)= & {\left[C x_{i}(t)+B \tilde{f}\left(x_{i}(t)\right)\right] d t+\tilde{g}\left(t, x_{i}(t)\right) d w(t) } \\
& +c \sum_{j=1}^{N} a_{i j} \Gamma \tilde{h}\left(x_{j}(t)\right) d t, \quad i=1,2, \ldots, N
\end{aligned}
$$

where $x_{i}(t)=\left(x_{i 1}(t), x_{i 2}(t), \ldots, x_{i n}(t)\right)^{T} \in \mathbb{R}^{n}$ is the state vector of the $i$-th node at time $t, C \in \mathbb{R}^{n \times n}, B \in \mathbb{R}^{n \times n}$, $w(t) \in \mathbb{R}^{m}$ is an $m$-dimensional Brownian motion, $\tilde{f}\left(x_{i}(t)\right)=$ $\left[\tilde{f}_{1}\left(x_{i 1}(t)\right), \tilde{f}_{2}\left(x_{i 2}(t)\right), \ldots, \tilde{f}_{n}\left(x_{i n}(t)\right)\right]^{T}$ satisfying $\tilde{f}(\mathbf{0})=\mathbf{0}$, $\tilde{g}: \mathbb{R}^{+} \times \mathbb{R}^{n} \rightarrow \mathbb{R}^{n \times m}$ is the noise intensity function matrix satisfying $\tilde{g}(t, \mathbf{0})=\mathbf{0}^{n \times m}$. The nonlinear function $\tilde{h}\left(x_{j}(t)\right)=$ $\left(\tilde{h}\left(x_{j 1}(t)\right), \tilde{h}\left(x_{j 2}(t)\right), \ldots, \tilde{h}\left(x_{j n}(t)\right)\right)^{T}$ satisfies the following conditions: $[(\tilde{h}(u)-\tilde{h}(v)) /(u-v)] \geq \vartheta>0$ for any $u, v \in \mathbb{R}$. The configuration coupling matrix $A=\left(a_{i j}\right)_{N \times N}$ is defined as follows: if there is a connection between node $i$ and node $j(j \neq i)$, then $a_{i j}=a_{j i}>0$, otherwise, $a_{i j}=a_{j i}=0$, and the diagonal elements are defined as $a_{i i}=-\sum_{j=1, j \neq i}^{N} a_{i j}$. $\Gamma=\operatorname{diag}\left\{\gamma_{1}, \gamma_{2}, \ldots, \gamma_{\mathrm{n}}\right\}>0$ is the inner coupling positive definite matrix between two connected nodes $i$ and $j$, and $c$ is the coupling strength of the network.
We have the following assumptions and lemma for the derivation of the main results.

Assumption 1: The nonlinear function $\tilde{f}(\cdot)$ is assumed to satisfy a Lipschitz condition, that is, there exists a constant $\kappa>0$ such that $\|\tilde{f}(u)-\tilde{f}(v)\| \leq \kappa\|u-v\|$ holds for any $u, v \in \mathbb{R}^{n}$.

Assumption 2: Assume that the noise intensity function matrix $g: \mathbb{R}^{+} \times \mathbb{R}^{n} \rightarrow \mathbb{R}^{n \times m}$ is uniformly Lipschitz continuous in terms of the norm induced by the trace inner product on the matrices

$$
\begin{array}{r}
\operatorname{trace}\left[(g(t, u)-g(t, v))^{T} \cdot(g(t, u)-g(t, v))\right] \\
\leq\|M(u-v)\|^{2} \quad \forall u, v \in \mathbb{R}^{n}
\end{array}
$$

where $M$ is a known constant matrix with compatible dimensions.

Lemma 1 ([30]): Consider the following stochastic system with impulses:

$\left\{\begin{array}{l}d x(t)=\phi(t, x(t)) d t+\eta(t, x(t)) d w(t), t \geq t_{0}, \quad t \neq t_{k}, \\ x\left(t_{k}^{+}\right)-x\left(t_{k}^{-}\right)=I_{k}\left(x\left(t_{k}^{-}\right)\right) \quad k \in \mathbb{N} .\end{array}\right.$

Assume that there exist a Lyapunov function $V(t, x(t))$, and functions $\varphi, \psi_{k}$ with $\varphi(t, 0)=\psi_{k}(0)=0$ for any $t \geq 0$, $k \in \mathbb{N}$, such that:

1) there exist positive constants $c_{1}$ and $c_{2}$ such that for all $t \geq t_{0}, c_{1}\|x(t)\| \leq V(t, x(t)) \leq c_{2}\|x(t)\| ;$

2) there exists continuous function $\varphi: \mathbb{R}^{+} \times \mathbb{R}^{+} \rightarrow \mathbb{R}$, and $\varphi(t, s)$ is concave on $s$ for each $t \in \mathbb{R}^{+}$, such that $\mathcal{L} V(t, x) \leq \varphi(t, V(t, x))$, where the operator $\mathcal{L}$ is defined as $\mathcal{L} V(t, x)=V_{t}(t, x)+V_{x}(t, x) \phi(t, x)+$ $(1 / 2) \operatorname{trace}\left[\eta^{T}(t, x) V_{x x} \eta(t, x)\right]$

3) there exist continuous and concave functions $\psi_{k}: \mathbb{R}^{+} \rightarrow$ $\mathbb{R}^{+}, k \in \mathbb{N}$, such that $V\left(t_{k}^{+}, x\left(t_{k}^{+}\right)\right) \leq \psi_{k}\left(V\left(t_{k}^{-}, x\left(t_{k}^{-}\right)\right)\right)$

then the exponential stability of the trivial solution of the following comparison systems:

$$
\left\{\begin{array}{l}
\dot{w}(t)=\varphi(t, w(t)), t \geq t_{0}, t \neq t_{k}, \\
w\left(t_{k}^{+}\right)=\psi_{k}\left(w\left(t_{k}^{-}\right)\right), \quad k \in \mathbb{N} \\
w\left(t_{0}\right)=E\left(V\left(t_{0}, x_{0}\right)\right)
\end{array}\right.
$$

implies the exponential stability of the trivial solution of the stochastic impulsive system (3).

Let $s(t)$ be a solution of an isolated node described by

$$
d s(t)=[C s(t)+B \tilde{f}(s(t))] d t+\tilde{g}(t, s(t)) d w(t)
$$

with initial condition $s_{0} \in \mathbb{R}^{n}$. In this paper, we want to control the nonlinear dynamical network (1) into the desired trajectory $s(t)$.

Let $e_{i}(t)=x_{i}(t)-s(t)$ be the error state of the node $i$. In order to force the whole network (1) into the desired trajectory $s(t)$, the following impulsive controllers are designed for $l$ nodes:

$$
I_{i}(t)=\left\{\begin{array}{l}
\sum_{k=1}^{+\infty} \mu e_{i}(t) \delta\left(t-t_{k}\right), i \in \mathfrak{D}\left(t_{k}\right), \# \mathfrak{D}\left(t_{k}\right)=l, \\
0, i \notin \mathfrak{D}\left(t_{k}\right)
\end{array}\right.
$$

where the constant $\mu \in(-2,0), \delta(\cdot)$ is the Dirac delta function, the time series $\left\{t_{1}, t_{2}, t_{3}, \ldots\right\}$ is a sequence of strictly increasing impulsive instants satisfying $\lim _{k \rightarrow \infty} t_{k}=+\infty$, 
and the index set of $\mathfrak{D}\left(t_{k}\right)$ is defined as follows: at time instant $t_{k}$, for the vectors $e_{1}\left(t_{k}\right), e_{2}\left(t_{k}\right), \ldots, e_{N}\left(t_{k}\right)$, one can reorder the states of the nodes such that $\left\|e_{p 1}\left(t_{k}\right)\right\| \geq\left\|e_{p 2}\left(t_{k}\right)\right\| \geq \cdots \geq$ $\left\|e_{p l}\left(t_{k}\right)\right\| \geq\left\|e_{p, l+1}\left(t_{k}\right)\right\| \geq \cdots \geq\left\|e_{p N}\left(t_{k}\right)\right\|$. Then the index set of $l$ controlled nodes $\mathfrak{D}\left(t_{k}\right)$ is defined as $\mathfrak{D}\left(t_{k}\right)=$ $\left\{p_{1}, p_{2}, \ldots, p_{l}\right\}$, and \#D $\left(t_{k}\right)=l$.

Since $c \sum_{j=1}^{N} a_{i j} \Gamma h(s(t))=0$, after adding the pinning impulsive controllers (6) to the dynamical network (1), one can obtain the following impulsively controlled dynamical network:

$$
\left\{\begin{aligned}
d e_{i}(t)= & {\left[C e_{i}(t)+B f\left(e_{i}(t)\right)\right] d t+g\left(t, e_{i}(t)\right) d w(t) } \\
& +c \sum_{j=1}^{N} a_{i j} \Gamma h\left(e_{j}(t)\right) d t, \quad t \neq t_{k}, k \in \mathbb{N}, \\
e_{i}\left(t_{k}^{+}\right)= & e_{i}\left(t_{k}^{-}\right)+\mu e_{i}\left(t_{k}^{-}\right), \quad i \in \mathfrak{D}\left(t_{k}\right), \# \mathfrak{D}\left(t_{k}\right)=l
\end{aligned}\right.
$$

where $f\left(e_{i}(t)\right)=\tilde{f}\left(x_{i}(t)\right)-\tilde{f}(s(t)), g\left(t, e_{i}(t)\right)=\tilde{g}\left(t, x_{i}(t)\right)$ $-\tilde{g}(t, s(t)), h\left(e_{i}(t)\right)=\tilde{h}\left(x_{i}(t)\right)-\tilde{h}(s(t))$. Since $[(\tilde{h}(u)-$ $\tilde{h}(v)) /(u-v)] \geq \vartheta>0$, we have $[(h(u)-h(v)) /(u-v)] \geq$ $\vartheta>0$ for any $u, v \in \mathbb{R}$.

Throughout this paper, we always assume that $e_{i}(t)$ is lefthand continuous at $t=t_{k}$, i.e., $e\left(t_{k}\right)=e\left(t_{k}^{-}\right)$. Therefore, the solutions of (7) are piecewise left-hand continuous functions with discontinuities at $t=t_{k}$ for $k \in \mathbb{N}$.

Definition 1: The trivial solution of the dynamical system (7) is said to be exponentially mean square stable if for any initial condition $e_{i}\left(t_{0}\right) \quad(i=1,2, \ldots, N)$, there exist positive constants $W_{0}$ and $\omega$ such that $E\left\{\sum_{i=1}^{N}\left\|x_{i}(t)\right\|^{2}\right\} \leq$ $W_{0} e^{-\omega\left(t-t_{0}\right)}$.

By referring to the concept of average dwell time [31], [32], a new concept named average impulsive interval has been proposed by the authors to describe wider class of impulsive signal, and has been utilized for the derivation of a unified synchronization criterion of dynamical networks in [7]. Since $\mu \in(-2,0)$, which means that the impulsive effects are stabilizing, the frequency of impulses should not be too low. In order to guarantee that the frequency of impulses is not too low, the following definition is presented.

Definition 2 ([7] average impulsive interval): The average impulsive interval of the impulsive sequence $\zeta=\left\{t_{1}, t_{2}, \ldots\right\}$ is less than $T_{a}$, if there exist a positive integer $N_{0}$ and a positive number $T_{a}$, such that

$$
N_{\zeta}(T, t) \geq \frac{T-t}{T_{a}}-N_{0} \quad \forall T \geq t \geq 0
$$

where $N_{\zeta}(T, t)$ denotes the number of impulsive times of the impulsive sequence $\zeta$ in the time interval $(t, T)$.

Remark 1: According to Definition 2, there is no strict requirement for the impulsive sequence on the upper bound of the impulsive intervals, which is normally necessary in the references concerning impulsive control. For very large $\varsigma>0$ and any $T_{a}>0$, many impulsive sequences $\left\{t_{1}, t_{2}, \ldots\right\}$ can be constructed such that the upper bound of the impulsive intervals is not less than to $\varsigma$ and simultaneously the average impulsive intervals are less than $T_{a}$. Let $k=\left\lceil\varsigma / T_{a}\right\rceil$ and $\epsilon>0$ very small. One simple example is $\zeta^{*}=\left\{t_{0}+T_{a}+\epsilon, t_{0}+\right.$ $T_{a}+2 \epsilon, \ldots, t_{0}+T_{a}+k \epsilon, t_{0}+T_{a}+k \epsilon+\varsigma, t_{0}+T_{a}+(k+$ 1) $\left.\epsilon+\varsigma, \ldots, t_{0}+T_{a}+2 k \epsilon+\varsigma, t_{0}+T_{a}+2 k \epsilon+2 \varsigma, \ldots\right\}$. For the impulsive sequence $\zeta^{*}$, the upper bound of the impulsive interval is $\varsigma$, which can be very large. Since the upper bound is used to represent the frequency of the impulsive sequence in [26], and [33]-[35], or identical impulsive interval is used in [36], the results obtained in these references are not available for the impulsive sequence $\zeta^{*}$ with very large upper bound of impulsive intervals, for which our results may be applicable.

Remark 2: By using the special example $\zeta^{*}$ presented in Remark 1, the idea behind this concept can be explained as follows: low-density impulses (such as " $t_{0}+T_{a}+k \epsilon, t_{0}+T_{a}+$ $k \epsilon+\varsigma$ ") are allowed to happen in a certain interval, and highdensity impulses (such as " $t_{0}+T_{a}+(k+1) \epsilon+\varsigma, \ldots, t_{0}+$ $T_{a}+2 k \epsilon+\varsigma$ ") should follow for compensation.

\section{MAIN RESULTS}

In this section, we will derive the main results about our pinning impulsive strategy for synchronization control of the stochastic dynamical network (1) with nonlinear coupling. Based on the above-mentioned assumptions and definitions, we can obtain the following theorem to show that the statecoupled dynamical network can be successfully stabilized to an objective state by only impulsive controlling a small fraction of nodes.

Theorem 1: Consider the controlled dynamical network (7)

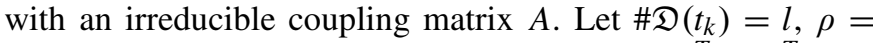
$1+(l / N) \cdot \mu(\mu+2) \in(0,1)$ and $\delta=\lambda_{\max }\left(C+C^{T}+M^{T} M\right)+$ $2 \sqrt{\lambda_{\max }\left(B^{T} B\right)} \kappa$. Suppose that Assumptions 1 and 2 hold, and the average impulsive interval of the impulsive sequence $\zeta=$ $\left\{t_{1}, t_{2}, \ldots\right\}$ is less than $T_{a}$. Then, the controlled dynamical network (7) is globally exponentially stable in mean square, if

$$
\frac{\ln \rho}{T_{a}}+\delta<0
$$

It means that the nonlinear stochastic dynamical network (1) can be exponentially controlled to the objective trajectory $s(t)$ by using pinning impulsive controllers (6).

Proof: Consider the following Lyapunov functions:

$$
V(t)=\sum_{i=1}^{N} e_{i}^{T}(t) e_{i}(t) .
$$

For $t \in\left(t_{k-1}, t_{k}\right], k \in \mathbb{N}$, we have

$$
\begin{aligned}
& \mathfrak{L} V(t) \\
= & 2 \sum_{i=1}^{N} e_{i}^{T}(t)\left[C e_{i}(t)+B f\left(e_{i}(t)\right)+c \sum_{j=1}^{N} a_{i j} \Gamma h\left(e_{j}(t)\right)\right] \\
& +\sum_{i=1}^{N} \operatorname{trace}\left[g^{T}\left(t, e_{i}(t)\right) g\left(t, e_{i}(t)\right)\right] \\
= & 2 \sum_{i=1}^{N}\left[e_{i}^{T}(t) C e_{i}(t)+e_{i}^{T}(t) B f\left(e_{i}(t)\right)\right] \\
& +2 c \sum_{i=1}^{N} \sum_{j=1}^{N} a_{i j} e_{i}^{T}(t) \Gamma h\left(e_{j}(t)\right) \\
& +\sum_{i=1}^{N} \operatorname{trace}\left[g^{T}\left(t, e_{i}(t)\right) g\left(t, e_{i}(t)\right)\right]
\end{aligned}
$$


By Assumptions 1 and 2, the following inequalities can be obtained:

$$
\begin{aligned}
& 2 e_{i}^{T}(t) B f\left(e_{i}(t)\right) \\
\leq & 2\left\|e_{i}(t)\right\| \cdot\left\|B f\left(e_{i}(t)\right)\right\| \\
\leq & 2\left\|e_{i}(t)\right\| \cdot \sqrt{\lambda_{\max }\left(B^{T} B\right)} \cdot\left\|f\left(e_{i}(t)\right)\right\| \\
\leq & 2 \sqrt{\lambda_{\max }\left(B^{T} B\right)} \kappa\left\|e_{i}(t)\right\|^{2} \\
= & 2 \sqrt{\lambda_{\max }\left(B^{T} B\right)} \kappa e_{i}^{T}(t) e_{i}(t)
\end{aligned}
$$

and

$$
\begin{aligned}
\operatorname{trace}\left[g^{T}\left(t, e_{i}(t)\right) g\left(t, e_{i}(t)\right)\right] & \leq\left\|M e_{i}(t)\right\|^{2} \\
& =e_{i}^{T}(t) M^{T} M e_{i}(t) .
\end{aligned}
$$

Since $[(h(u)-h(v)) /(u-v)] \geq \vartheta>0$, it follows from the diffusive property of symmetric matrix $A$ that

$$
\begin{aligned}
& 2 c \sum_{i=1}^{N} \sum_{j=1}^{N} a_{i j} e_{i}^{T}(t) \Gamma h\left(e_{j}(t)\right) \\
= & 2 c \sum_{i=1}^{N} \sum_{j=1}^{N} a_{i j}\left[\sum_{\theta=1}^{n} e_{i \theta}(t) \gamma_{\theta} h\left(e_{j \theta}(t)\right)\right] \\
= & 2 c \sum_{\theta=1}^{n} \gamma_{\theta}\left[\sum_{i=1}^{N} \sum_{j=1}^{N} e_{i \theta}(t) a_{i j} h\left(e_{j \theta}(t)\right)\right] \\
= & c \sum_{\theta=1}^{n} \gamma_{\theta} \sum_{i=1}^{N} \sum_{\substack{j=1 \\
j \neq i}}^{N} a_{i j}\left(e_{i \theta}(t)-e_{j \theta}(t)\right) \\
& \times\left(h\left(e_{i \theta}(t)\right)-h\left(e_{j \theta}(t)\right)\right) \\
\leq & -c \sum_{\theta=1}^{n} \sum_{i=1}^{N} \sum_{\substack{j=1 \\
j \neq i}}^{N} \vartheta \gamma_{\theta} a_{i j}\left(e_{i \theta}(t)-e_{j \theta}(t)\right)^{2} \\
\leq & 0 .
\end{aligned}
$$

Considering (12)-(14), it follows from (11) that

$$
\begin{aligned}
& \mathcal{L} V(t) \\
\leq & \sum_{i=1}^{N} e_{i}^{T}(t)\left[C+C^{T}+M^{T} M\right] e_{i}(t) \\
& +\sum_{i=1}^{N} 2 \sqrt{\lambda_{\max }\left(B^{T} B\right)} \kappa e_{i}^{T}(t) e_{i}(t) \\
\leq & \left(\lambda_{\max }\left(C+C^{T}+M^{T} M\right)+2 \sqrt{\lambda_{\max }\left(B^{T} B\right)} \kappa\right) \\
& \times \sum_{i=1}^{N} e_{i}^{T}(t) e_{i}(t) \\
= & \delta \cdot V(t), \quad \text { for } t \in\left(t_{k-1}, t_{k}\right], \quad k \in \mathbb{N} .
\end{aligned}
$$

For any $k \in \mathbb{N}$, let $\alpha\left(t_{k}\right)=\min \left\{\left\|e_{i}\left(t_{k}\right)\right\|: i \in \mathfrak{D}\left(t_{k}\right)\right\}$ and $\beta\left(t_{k}\right)=\max \left\{\left\|e_{i}\left(t_{k}\right)\right\|: i \notin \mathfrak{D}\left(t_{k}\right)\right\}$. According to the selection of nodes in set $\mathfrak{D}\left(t_{k}\right)$, we have $\alpha\left(t_{k}\right) \geq \beta\left(t_{k}\right)$. Since $\rho=$ $1+(l / N) \cdot \mu(\mu+2) \in(0,1)$, we get $(1-\rho)(N-l)=$ $\left[\rho-(1+\mu)^{2}\right] l$. Hence, one has

$$
\begin{aligned}
& (1-\rho) \sum_{i \notin \mathfrak{D}\left(t_{k}\right)} e_{i}^{T}\left(t_{k}^{-}\right) e_{i}\left(t_{k}^{-}\right) \\
\leq & (1-\rho)(N-l)\left(\beta\left(t_{k}\right)\right)^{2} \\
\leq & (1-\rho)(N-l)\left(\alpha\left(t_{k}\right)\right)^{2} \\
\leq & l\left[\rho-(1+\mu)^{2}\right]\left(\alpha\left(t_{k}\right)\right)^{2} \\
\leq & {\left[\rho-(1+\mu)^{2}\right] \sum_{i \in \mathfrak{D}\left(t_{k}\right)} e_{i}^{T}\left(t_{k}^{-}\right) e_{i}\left(t_{k}^{-}\right) }
\end{aligned}
$$

which follows that

$$
\begin{aligned}
(1+\mu)^{2} \sum_{i \in \mathfrak{D}\left(t_{k}\right)} e_{i}^{T}\left(t_{k}^{-}\right) e_{i}\left(t_{k}^{-}\right) & +\sum_{i \notin \mathfrak{D}\left(t_{k}\right)} e_{i}^{T}\left(t_{k}^{-}\right) e_{i}\left(t_{k}^{-}\right) \\
& \leq \rho \sum_{i=1}^{N} e_{i}^{T}\left(t_{k}^{-}\right) e_{i}\left(t_{k}^{-}\right) .
\end{aligned}
$$

Then, for any $k \in \mathbb{N}$, we yield

$$
\begin{aligned}
& V\left(t_{k}^{+}\right) \\
= & \sum_{i=1}^{N} e_{i}^{T}\left(t_{k}^{+}\right) e_{i}\left(t_{k}^{+}\right) \\
= & \sum_{i \in \mathfrak{D}\left(t_{k}\right)} e_{i}^{T}\left(t_{k}^{+}\right) e_{i}\left(t_{k}^{+}\right)+\sum_{i \notin \mathfrak{D}\left(t_{k}\right)} e_{i}^{T}\left(t_{k}^{+}\right) e_{i}\left(t_{k}^{+}\right) \\
= & \sum_{i \in \mathfrak{D}\left(t_{k}\right)}(1+\mu)^{2} e_{i}^{T}\left(t_{k}^{-}\right) e_{i}\left(t_{k}^{-}\right)+\sum_{i \notin \mathfrak{D}\left(t_{k}\right)} e_{i}^{T}\left(t_{k}^{-}\right) e_{i}\left(t_{k}^{-}\right) \\
\leq & \rho \sum_{i=1}^{N} e_{i}^{T}\left(t_{k}^{-}\right) e_{i}\left(t_{k}^{-}\right) \\
= & \rho V\left(t_{k}^{-}\right) .
\end{aligned}
$$

By (15) and (18), we can obtain the following comparison system (19) for the controlled dynamical network (7):

$$
\left\{\begin{array}{l}
\dot{w}(t)=\delta w(t), t \geq t_{0}, t \neq t_{k}, \\
w\left(t_{k}^{+}\right)=\rho w\left(t_{k}^{-}\right), \quad \rho \in(0,1), k \in \mathbb{N}, \\
w\left(t_{0}\right)=E\left(V\left(t_{0}\right)\right) .
\end{array}\right.
$$

According to (19), for any $t \in \mathbb{R}^{+}$, one has

$$
w(t)=E\left(V\left(t_{0}\right)\right) \cdot e^{\delta\left(t-t_{0}\right)} \rho^{N_{\zeta}\left(t, t_{0}\right)}
$$

where $N_{\zeta}\left(t, t_{0}\right)$ means the number of impulses of the impulsive sequence $\zeta$ in the time interval $\left(t_{0}, t\right)$.

According to the facts that $\rho \in(0,1)$ and that the average impulsive interval of the impulsive sequence $\zeta=\left\{t_{1}, t_{2}, \ldots\right\}$ is less than $T_{a}$, it follows from Definition 2 that

$$
\begin{aligned}
w(t) & =E\left(V\left(t_{0}\right)\right) \cdot e^{\delta\left(t-t_{0}\right)} \rho^{N_{\zeta}\left(t, t_{0}\right)} \\
& \leq E\left(V\left(t_{0}\right)\right) \cdot e^{\delta\left(t-t_{0}\right)} \rho^{\frac{t-t_{0}}{T_{a}}-N_{0}} \\
& =E\left(V\left(t_{0}\right)\right) \rho^{-N_{0}} \cdot e^{\delta\left(t-t_{0}\right)} \cdot e^{\frac{\ln \rho}{T_{a}}\left(t-t_{0}\right)} \\
& =E\left(V\left(t_{0}\right)\right) \rho^{-N_{0}} \cdot e^{\left(\frac{\ln \rho}{T_{a}}+\delta\right)\left(t-t_{0}\right)} .
\end{aligned}
$$

Since $\left(\ln \rho / T_{a}\right)+\delta<0$, the trivial solution of the comparison system (19) is exponentially stable. By Lemma 1, we can conclude that the controlled dynamical network (7) is exponential stable, which further implies that the dynamical network (1) can be exponentially stabilized to the objective 
trajectory $s(t)$ by only impulsively controlling a small fraction of nodes. Theorem 1 is proved.

Remark 3: By using the Lyapunov method combined with the comparison principle, the exponential stability criterion of the pinning impulsively controlled dynamical network has been obtained. It means that the state-coupled dynamical network can be efficiently forced to the objective trajectory by using pinning impulsive controllers. Our result displays another kind of effective and relatively cheap control strategy for the synchronization of complex dynamical networks.

Remark 4: The criterion presented in Theorem 1 is closely related to the system parameters, average impulsive interval, impulsive strength, and the proportion of the controlled nodes. The criterion can be easily judged without large computation. In the following, a theorem will be given to explicitly show how many nodes should be controlled for a successful synchronization control of the nonlinear stochastic dynamical network (1).

Theorem 2: Consider the controlled dynamical network (7) with an irreducible coupling matrix $A$. Let $\delta=\lambda_{\max }\left(C+C^{T}+\right.$ $\left.M^{T} M\right)+2 \sqrt{\lambda_{\max }\left(B^{T} B\right)} \kappa$. Suppose that Assumptions 1 and 2 hold, and the average impulsive interval of the impulsive sequence $\zeta=\left\{t_{1}, t_{2}, \ldots\right\}$ is less than $T_{a}$. Then, the controlled dynamical network (7) is globally exponentially stable in mean square, if

$$
\frac{l}{N}>\frac{1}{\mu(\mu+2)}\left(e^{-\delta T_{a}}-1\right)
$$

where $l \ll N$ is the number of nodes to be controlled.

Proof: Since $\rho=1+(l / N) \cdot \mu(\mu+2) \in(0,1)$, this theorem can be proved by using Theorem 1 . The detailed proof is omitted here.

Remark 5: Since $l \ll N$ is the number of nodes to be controlled, $(l / N) \ll 1$ is the proportion of the controlled nodes. The numerical example illustrates that the stochastic dynamical network can be successfully synchronized to a certain objective trajectory by impulsively controlling $10 \%$ of the nodes. It means that our pinning impulsive strategy is effective for the synchronization of networks with a small fraction of nodes controlled.

Remark 6: Similar with Theorem 2, we can conclude that: the stochastic dynamical network (1) can be forced to the objective trajectory $s(t)$ by pinning impulsive controllers (6) if one of the following inequalities is satisfied:

$$
\begin{aligned}
& \text { 1) } T_{a}<-\frac{1}{\delta} \ln \left(\mu(\mu+2) \cdot \frac{l}{N}+1\right) \text {; } \\
& \text { 2) }-\sqrt{\frac{e^{-\delta T_{a}}-1}{l / N}+1}-1<\mu<\sqrt{\frac{e^{-\delta T_{a}-1}}{l / N}+1}-1 .
\end{aligned}
$$

Remark 7: The synchronization problem for discrete-time stochastic dynamical networks has drawn much research attention [36], [37]. Our pinning impulsive strategy obtained in this paper is also applicable to the case of discrete-time dynamical networks.

\section{NumERICAL EXAMPLE}

In this section, numerical example will be given to demonstrate the effectiveness of our main results. A chaotic system with Brownian noise is selected as the isolated node of the

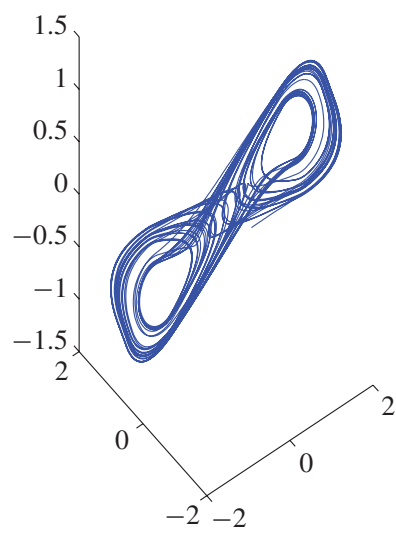

(a)

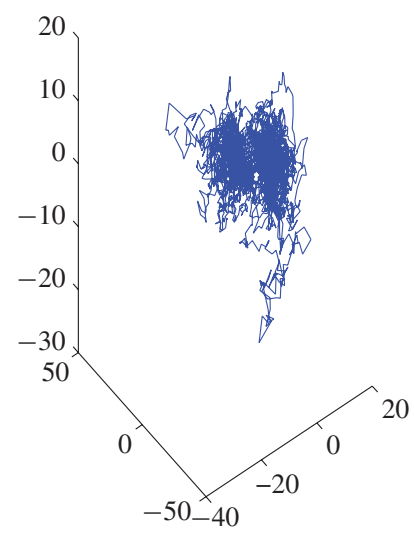

(b)
Fig. 1. Phase trajectories of single dynamical system (a) without noise and (b) with noise.

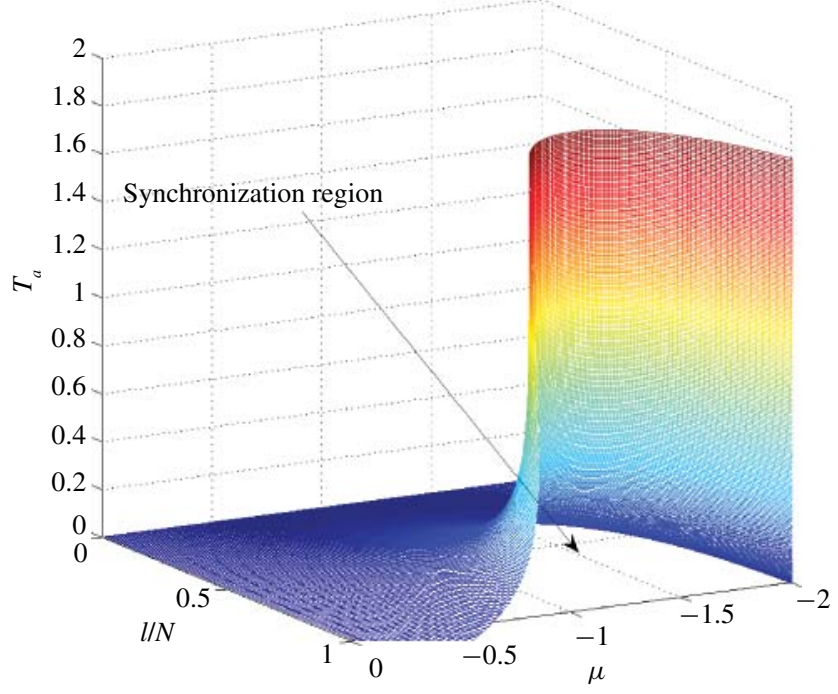

Fig. 2. Estimation of boundaries of synchronization region with respect to $\mu, T_{a}$, and $(l / N)$

dynamical network, and the $i$-th node is described as follows:

$$
d x_{i}(t)=\left[C x_{i}(t)+B \tilde{f}\left(x_{i}(t)\right)\right] d t+\tilde{g}\left(t, x_{i}(t)\right) d w_{i}(t)
$$

where $x_{i}(t)=\left(x_{i 1}(t), x_{i 2}(t), x_{i 3}(t)\right)^{T} \in \mathbb{R}^{3}, d w_{i}(t)$ is an 3-D Brownian motion, and the parameters are given as $C=$ $-1.2 \cdot I_{3}, B=\left(\begin{array}{rrr}1.16 & -1.5 & -1.5 \\ -1.5 & 1.16 & -2.0 \\ -1.2 & 2.0 & 1.16\end{array}\right)$, nonlinear function $\tilde{f}\left(x_{i}(t)\right)=\left(\tanh \left(x_{i 1}\right), \tanh \left(x_{i 2}\right), \tanh \left(x_{i 3}\right)\right)^{T}$, and the noise intensity function matrix $\tilde{g}\left(t, x_{i}(t)\right)=0.5 \cdot\left\|x_{i}(t)\right\| \cdot I_{3}$. Then we have $\kappa=1$ and $M=0.5 \cdot I_{3}$ for Assumptions 1 and 2 . System (23) without Brownian motion noise has a chaotic attractor [38] with initial value $[0.3,-0.1,-0.4]$ as shown in Fig. 1.

In this example, a Newman-Watts small-world network with 100 nodes will be considered [39]. The small-world network is generated by taking initial neighboring nodes $k=4$ and the edge adding probability $p=0.1$. The coupling matrix $A$ is defined as follows: if there is a connection between nodes $i$ and $j$, then $a_{i j}=a_{j i}=1$, otherwise $a_{i j}=a_{j i}=0$. The nonlinear coupling function $\tilde{h}$ is taken as $\tilde{h}(z)=z+\tanh (z)$ 


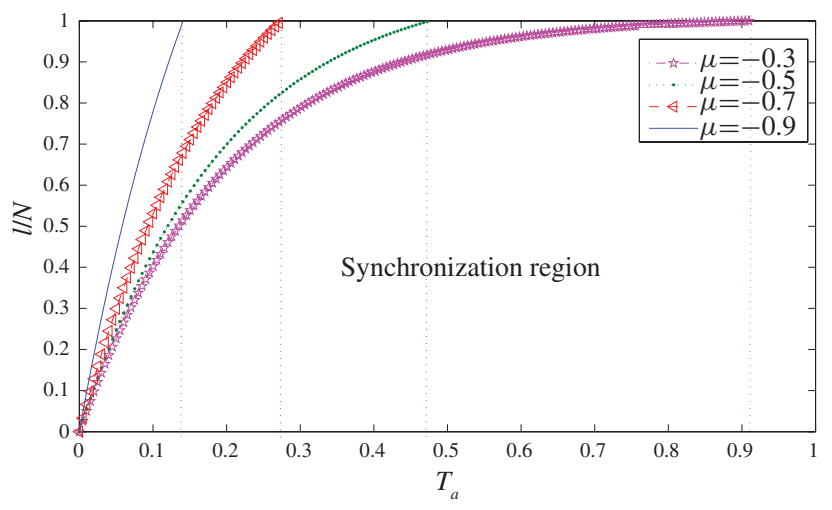

Fig. 3. Estimation of the synchronization region about $T_{a}$ and $(l / N)$ with different $\mu$.

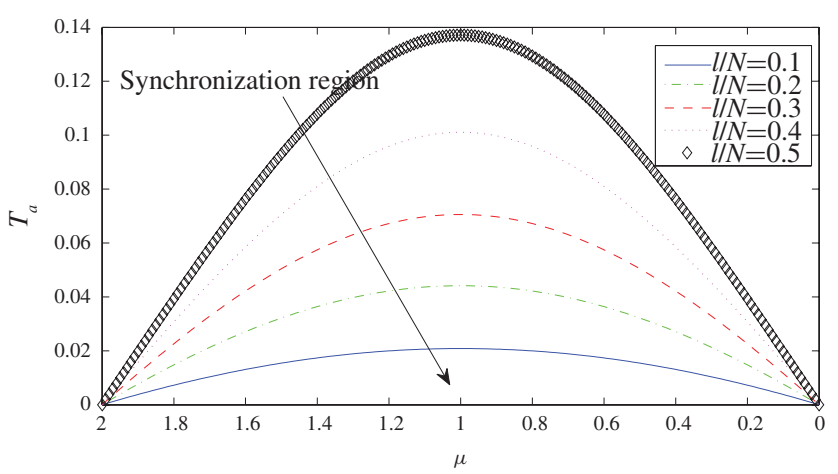

Fig. 4. Estimation of the synchronization region about $\mu$ and $T_{a}$ with different $(l / N)$.

with $\vartheta=1$, coupling strength $c=1$ and the inner coupling matrix $\Gamma=I_{3}$. By some simple calculations, we can obtain that $\delta=5.0542$. Theorem 2 and Remark 6 have been given to show the explicit relationship between three quantities $(l / N)$, $\mu$ and $T_{a}$. Fig. 2 shows the synchronization region of the controlled dynamical network for $\mu, T_{a}$, and $(l / N)$. Fig. 3 displays the estimation of boundaries of the synchronization regions for different $\mu$ with respect to $T_{a}$ and $(l / N)$.

Remark 8: According to the property of the impulsive control, one knows that if $\mu=-1$, the error state of the controlled system becomes zero immediately after the impulsive controller. Hence the impulsive interval can be $+\infty$ when $\mu=-1$ [40], [41]. However, since only a small fraction of nodes is controlled at each impulsive instant $t_{k}$, the error states of the controlled nodes would become nonzero due to the interconnections with some other uncontrolled nodes even for $\mu=-1$. Therefore, when $\mu=-1$, the average impulsive interval has an upper bound as shown in Fig. 4. In some real applications, one may choose $\mu=-1$ to have the maximum impulsive interval.

Now, we take special values of $\mu, T_{a}$, and $(l / N)$ for numerical illustration. Let $\mu=-0.9$ and $T_{a}=0.02$, one can get that $[1 / \mu(\mu+2)]\left(e^{-\delta T_{a}}-1\right)=0.0971$. By Theorem 2 , it can be concluded that the nonlinear stochastic dynamical network can be synchronized to the objective trajectory if $(l / N)=10 \%$ of the nodes is controlled. In other words, ten nodes should be impulsively controlled in the generated small-world network containing 100 nodes. The trivial point $s(t)=0$ is taken as the objective trajectory in this example.

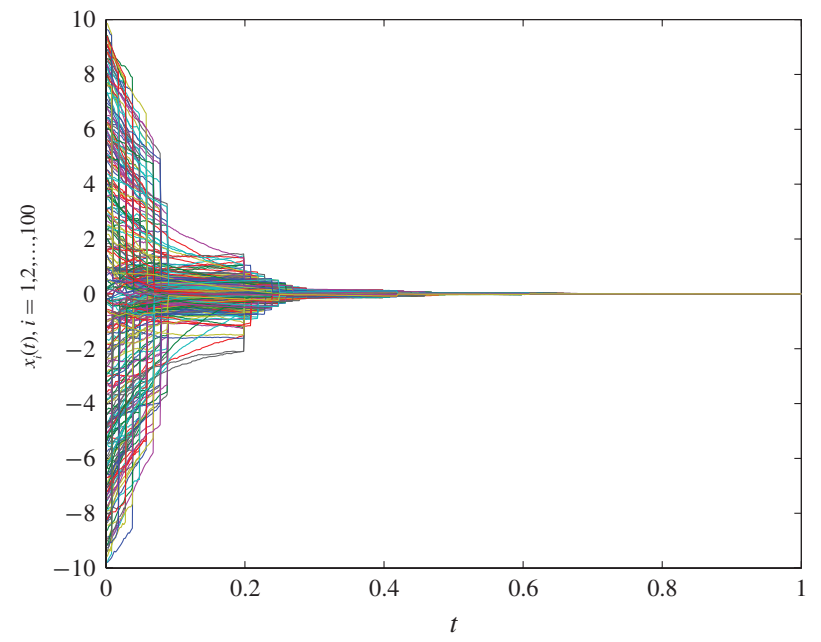

Fig. 5. Pinning impulsive synchronization of small-world coupled dynamical networks by controlling $10 \%$ nodes.

Fig. 5 presents the numerical process for the synchronization control, in which the impulsive sequence is generated with $N_{0}=10$ and $\epsilon=0.01$. All initial values of the dynamical network are uniformly randomly selected from $[-10,10]$.

\section{CONCLUSION}

In this paper, the synchronization control problem of stochastic dynamical networks with nonlinear coupling has been studied by pinning a small fraction of nodes with impulsive controllers. The uncontrolled nodes can be virtually forced to the desired synchronization trajectory by the pinned nodes via the inter-connections. Some stability criteria have been established to guarantee the success of synchronization via pinning controllers, and moreover the stable region can be explicitly revealed and plotted. Numerical examples are also given to demonstrate the effectiveness of our proposed control strategy.

\section{REFERENCES}

[1] S. Strogatz, "Exploring complex networks," Nature, vol. 410, pp. 268276, Mar. 2001.

[2] M. Newmann, "The structure and function of complex networks," SIAM Rev., vol. 45, no. 2, pp. 167-256, 2003.

[3] A. Arenas, A. Díaz-Guilera, J. Kurths, Y. Moreno, and C. Zhou, "Synchronization in complex networks," Phys. Rep., vol. 469, no. 3, pp. 93-153, 2008.

[4] D. Watts and S. Strogatz, "Collective dynamics of 'small-world' networks," Nature, vol. 393, no. 6684, pp. 440-442, 1998.

[5] X. F. Wang, "Complex networks: Topology, dynamics and synchronization,” Int. J. Bifurcation Chaos, vol. 12, no. 5, pp. 885-916, 2002.

[6] A. Barabási and R. Albert, "Emergence of scaling in random networks," Science, vol. 286, no. 5439, pp. 509-512, 1999.

[7] J. Lu, D. Ho, and J. Cao, "A unified synchronization criterion for impulsive dynamical networks," Automatica, vol. 46, no. 7, pp. 12151221, Jul. 2010.

[8] J. Lu, D. Ho, and J. Kurths, "Consensus over directed static networks with arbitrary communication delays," Phys. Rev. E, vol. 80, no. 6, pp. 066121-1-066121-7, 2009.

[9] A. Motter, C. Zhou, and J. Kurths, "Network synchronization, diffusion, and the paradox of heterogeneity," J. Phys. Rev. E, vol. 71, no. 1, pp. 016116-1-016116-9, 2005.

[10] H. Gao, J. Lam, and G. Chen, "New criteria for synchronization stability of general complex dynamical networks with coupling delays," Phys. Lett. A, vol. 360, no. 2, pp. 263-273, 2006.

[11] J. Cao, G. Chen, and P. Li, "Global synchronization in an array of delayed neural networks with hybrid coupling," IEEE Trans. Syst. Man Cybern. Part B, vol. 38, no. 2, pp. 488-498, Apr. 2008. 
[12] J. Lu, D. Ho, J. Cao, and J. Kurths, "Exponential synchronization of linearly coupled neural networks with impulsive disturbances," IEEE Trans. Neural Netw., vol. 22, no. 2, pp. 329-335, Feb. 2011.

[13] J. Lü and G. Chen, "A time-varying complex dynamical network model and its controlled synchronization criteria," IEEE Trans. Automat. Control, vol. 50, no. 6, pp. 841-846, Jun. 2005.

[14] C. Wu, Synchronization in Complex Networks of Nonlinear Dynamical Systems. Singapore: World Scientific, 2007.

[15] C. Zhou, A. Motter, and J. Kurths, "Universality in the synchronization of weighted random networks," Phys. Rev. Lett., vol. 96, no. 3, pp. 034101-1-034101-4, 2006.

[16] V. Vaidhyanathan, Regulation and Control Mechanisms in Biological Systems. Englewood Cliffs, NJ: Prentice-Hall, 1993.

[17] N. Koshiya and J. Smith, "Neuronal pacemaker for breathing visualized in vitro," Nature, vol. 400, pp. 360-363, May 1999.

[18] X. Wang and G. Chen, "Pinning control of scale-free dynamical networks," Phys. A, vol. 310, nos. 3-4, pp. 521-531, 2002.

[19] X. Li, X. Wang, and G. Chen, "Pinning a complex dynamical network to its equilibrium," IEEE Trans. Circuits Syst.-I, vol. 51, no. 10, pp. 2074 2087, Oct. 2004.

[20] T. Chen, X. Liu, and W. Lu, "Pinning complex networks by a single controller," IEEE Trans. Circuits Syst.-I, vol. 54, no. 6, pp. 1317-1326, Jun. 2007.

[21] J. Lu, D. Ho, and L. Wu, "Exponential stabilization in switched stochastic dynamical networks," Nonlinearity, vol. 22, no. 4, pp. 889 911, 2009

[22] J. Lu, D. Ho, and Z. Wang, "Pinning stabilization of linearly coupled stochastic neural networks via minimum number of controllers," IEEE Trans. Neural Netw., vol. 20, no. 10, pp. 1617-1629, Oct. 2009.

[23] J. Zhou, J. Lu, and J. Lu, "Adaptive synchronization of an uncertain complex dynamical network," IEEE Trans. Automat. Control, vol. 51, no. 4, pp. 652-656, Apr. 2006.

[24] J. Lu, D. Ho, and J. Cao, "Synchronization in an array of nonlinearly coupled chaotic neural networks with delay coupling," Int. J. Bifurcation Chaos, vol. 18, no. 10, pp. 3101-3111, 2008.

[25] N. Mahdavi, M. Menhaj, J. Kurths, J. Lu, and A. Afshar, "Pinning impulsive synchronization of complex dynamical networks," Int. J. Bifurcation Chaos, 2011, to be published.

[26] Z. Guan, Z. Liu, G. Feng, and Y. Wang, "Synchronization of complex dynamical networks with time-varying delays via impulsive distributed control," IEEE Trans. Circuits Syst. I: Regular Papers, vol. 57, no. 8, pp. 2182-2195, Aug. 2010.

[27] X. Yang, J. Cao, and J. Lu, "Synchronization of delayed complex dynamical networks with impulsive and stochastic effects," Nonlinear Anal.: Real World Applicat., vol. 12, no. 4, pp. 2252-2266, 2011.

[28] G. Hu and Z. Qu, "Controlling spatiotemporal chaos in coupled map lattice systems," Phys. Rev. Lett., vol. 72, no. 1, pp. 68-71, 1994.

[29] Z. Guan, D. Hill, and X. Shen, "On hybrid impulsive and switching systems and application to nonlinear control," IEEE Trans. Automat. Control, vol. 50, no. 7, pp. 1058-1062, Jul. 2005.

[30] B. Liu, "Stability of solutions for stochastic impulsive systems via comparison approach," IEEE Trans. Automat. Control, vol. 53, no. 9 , pp. 2128-2133, Oct. 2008.

[31] J. P. Hespanha and A. S. Morse, "Stability of switched systems with average dwell-time," in Proc. IEEE 38th Conf. Decision Control, Phoenix, AZ, Dec. 1999, pp. 2655-2660.

[32] D. Liberzon, Switching in Systems and Control. New York: SpringerVerlag, 2003.

[33] Q. Zhang and J. Lu, "Impulsively control complex networks with different dynamical nodes to its trivial equilibrium," Comput. Math. Applicat., vol. 57, no. 7, pp. 1073-1079, Apr. 2009.

[34] H. Zhang, Z. Guan, and D. Ho, "On synchronization of hybrid switching and impulsive networks," in Proc. IEEE 45th Conf. Decision Control, San Diego, CA, Dec. 2006, pp. 2765-2770.

[35] B. Liu, K. Teo, and X. Liu, "Robust exponential stabilization for largescale uncertain impulsive systems with coupling time-delays," Nonlinear Anal., vol. 68, no. 5, pp. 1169-1183, 2008.

[36] H. Zhang, T. Ma, G. Huang, and Z. Wang, "Robust global exponential synchronization of uncertain chaotic delayed neural networks via dualstage impulsive control," IEEE Trans. Syst. Man Cybern. Part B, vol. 40 , no. 3, pp. 831-844, Jun. 2010.

[37] B. Shen, Z. Wang, and X. Liu, "Bounded $H_{\infty}$ synchronization and state estimation for discrete time-varying stochastic complex networks over a finite-horizon," IEEE Trans. Neural Netw., vol. 22, no. 1, pp. 145-157, Jan. 2011.
[38] F. Zou and J. Nossek, "Bifurcation and chaos in cellular neural networks," IEEE Trans. Circuits Syst.-I, vol. 40, no. 3, pp. 166-173, Mar. 1993.

[39] M. Newman and D. Watts, "Scaling and percolation in the small-world network model," Phys. Rev. E, vol. 60, no. 6, pp. 7332-7342, 1999.

[40] T. Yang, Impulsive Systems and Control: Theory and Application. New York: Nova Science, 2001

[41] M. Hu, Y. Yang, and Z. Xu, "Impulsive control of projective synchronization in chaotic systems," Phys. Lett. A, vol. 372 , no. 18, pp. 3228 3233, Apr. 2008.

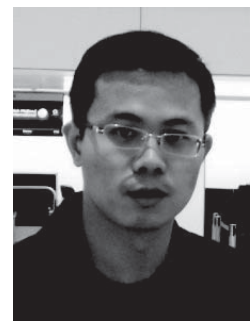

Jianquan Lu received the B.S. degree in mathematics from Zhejiang Normal University, Zhejiang, China, the M.S. degree in mathematics from Southeast University, Nanjing, China, and the Ph.D. degree from the City University of Hong Kong, Kowloon, Hong Kong, in 2003, 2006, and 2009, respectively.

$\mathrm{He}$ is currently an Associate Professor with the Department of Mathematics, Southeast University. He has published over 25 papers in refereed international journals. His current research interests include collective behavior in complex dynamical networks, multi-agent systems, hybrid systems, synchronization, nonlinear systems and controls, and neural networks.

Dr. Lu is an Associate Editor of Neural Processing Letters (Springer) and a Guest Editor of Mathematics and Computers in Simulation (Elsevier). He is the recipient of the Jiangsu Provincial Outstanding Master's Thesis Award in 2007, Top 100 Most Cited Chinese Papers Published in International Journals in 2007, an Alexander von Humboldt Fellowship in 2010, a Program for New Century Excellent Talents in University by the Ministry of Education, China, in 2010, and the First Award of Jiangsu Provincial Progress in Science and Technology as the Second Project Member in 2010.

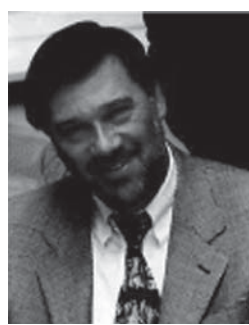

Jürgen Kurths received the degree in mathematics from the University of Rostock, Rostock, Germany, and the Ph.D. and Dr.Habil. degrees from the GDR Academy of Sciences, Berlin, Germany in 1983 and 1990, respectively.

$\mathrm{He}$ was a Full Professor with the University of Potsdam, Potsdam, Germany, from 1994 to 2008. Currently, he is a Professor of nonlinear dynamics with Humboldt University, Berlin, Germany, and the Chair of the research domain transdisciplinary concepts of the Potsdam Institute for Climate Impact Research. He has published more than 450 papers and two monographs which are cited more than 16000 times. His current research interests include complex synchronization phenomena, complex networks, time series analysis and their applications in climatology, sustainability, physiology, and engineering.

He is a fellow of the American Physical Society and the Fraunhofer Society in Germany, and a member of Academia Europaea. He received a HumboldtCSIR Research Prize in 2005 and a Dr.h.c. in 2008. He is on the editorial boards of more than ten journals, among them CHAOS, Philosophical Trans. Royal Soc. A, European Journal of Physics ST, and Nonlinear Processes in Geophysics. 


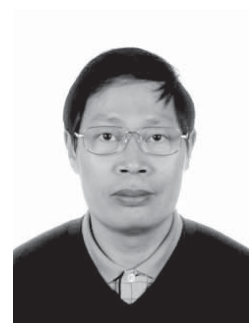

Jinde Cao (M'07-SM'07) received the B.S. degree from Anhui Normal University, Wuhu, China, the M.S. degree from Yunnan University, Kunming, China, and the Ph.D. degree from Sichuan University, Chengdu, China, in 1986, 1989, and 1998 , respectively, all in mathematics/applied mathematics.

He was with Yunnan University from 1989 to 2000. In 2000, he joined the Department of Mathematics, Southeast University, Nanjing, China. From 2001 to 2002, he was a Post-Doctoral Research Fellow with the Department of Automation and Computer-Aided Engineering, Chinese University of Hong Kong, Shatin, Hong Kong. From 2006 to 2008, he was a Visiting Research Fellow and a Visiting Professor with the School of Information Systems, Computing and Mathematics, Brunel University, Middlesex, U.K. He is the author or co-author of more than 160 research papers and five edited books. His current research interests include nonlinear systems, neural networks, complex systems, complex networks, stability theory, and applied mathematics.

Dr. Cao was an Associate Editor of the IEEE Transactions on NEURAL NETWORKS from 2006 to 2009. He is an Associate Editor of the Journal of the Franklin Institute, Mathematics and Computers in Simulation, Neurocomputing, Discrete Dynamics in Nature and Society, the International Journal of Differential Equations, and Differential Equations and Dynamical Systems. $\mathrm{He}$ is a reviewer of Mathematical Reviews and Zentralblatt-Math.

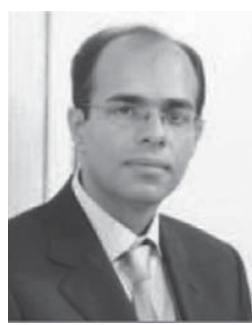

Nariman Mahdavi received the B.Sc. degree from the Isfahan University of Technology, Isfahan, Iran, in 2004, and the M.Sc. (first class) and Ph.D. degrees from the Amirkabir University of Technology, Tehran, Iran, in 2007 and 2011, respectively, all in electrical engineering.

He was a Guest Researcher with the Potsdam Institute for Climate Impact Research, Potsdam, Germany, from August 2010 to February 2011. His current research interests include complex systems and their synchronization, adaptive and impulsive controls, computational intelligence, and their applications in controls.

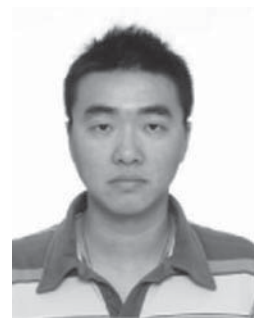

Chi Huang received the M.S. degree in mathematics from the City University of Hong Kong, Kowloon, Hong Kong, in 2009. He is currently pursuing the Ph.D. degree with the Department of Mathematics, City University of Hong Kong.

His current research interests include multiagent systems, complex dynamical networks, and sensor networks.

Mr. Huang was the recipient of the Best Paper Award in the Eighth Asian Control Conference in 2011. 